\title{
Donación y Subjetividad en La Nueva Fenomenología en Francia: Marion, Romano y Barbaras ${ }^{\mathrm{I}}$
}

\author{
Patricio Mena Malet
}

\begin{abstract}
Resumen: El siguiente trabajo tiene por objetivo examinar la Nueva fenomenología en Francia, a través de las obras de Marion, Romano y Barbaras. Nuestra hipótesis de lectura es que estas fenomenologías, por un lado la de Marion y Romano, y por el otro la de Barbaras, coinciden en dos cuestiones: 1) el privilegio que otorgan a la donación y, 2) la necesidad de pensar la subjetividad abandonando el paradigma de la subjetividad trascendental husserliano. Ambas propuestas requieren de un examen de las intenciones metódicas de la fenomenología husserliana y es, en dicha discusión, que ganan, al mismo tiempo, la originalidad de su marcha fenomenológica.
\end{abstract}

Palabras Claves: Subjetividad. Donación. Deseo. Acontecimiento. Fenómeno.

1. No cabe duda que la fenomenología en Francia es actualmente muy fructífera. Obras tan importantes como las de Renaud Barbaras, JeanLuc Marion, Michel Henry, Henry Maldiney, Françoise Dastur, Jean-Louis Chrétien, Jean-Yves Lacoste, Claude Romano, Emmanuel Housset, Jocelyn Benoist, Bruce Bégout, entre otros, dinamizan la escena de la filosofía actual con propuestas originales que actualmente son debatidas y discutidas. Ciertamente, en los últimos 30 años en Francia, la fenomenología se ha desarrollado de manera muy importante, tal como lo han señalado en un estudio reciente Lásló Tengelyi y Hans-Dieter Gondek (2011). Es a partir de 1990, que las obras de estos autores toma el relevo de las de Sartre, Merleau-Ponty y Levinas, tras un largo silencio de la fenomenología que cedió su lugar a movimientos como el estructuralismo y las filosofías de la diferencia y la deconstrucción. Sin embargo, aquellos años de silencio no significaron un abandono de la fenomenología, pues fueron verdaderos años de formación marcados por la publicación de los inéditos de Husserl que abría otras vías de interrogación fenomenológica. No cabe duda, entonces, que la fenomenología, que ha sido revivificada a partir de la obra paciente de Jean-Luc Marion - por nombrar solo a este autor-, según el juicio de Tengelyi y Gondek, haya tomado en

${ }^{1}$ Este artículo ha sido escrito en el marco del proyecto Fondecyt Regular No 1140997 (2014-2016), del que el autor es el Investigador Responsable.

http://dx.doi.org/10.1590/S0101-31732017000100010

2 Departamento de Ciencias Sociales. Universidad de La Frontera. Temuco, Chile. E-mail: patricio. mena.m@ufrontera.cl 
Francia "el rol de la filosofía" (MARION, 2004, p. 7). Basta leer los múltiples comentarios de Jean Greisch (2009) para reconocer el lugar predominante y la originalidad con la que se ha desarrollado la fenomenología en Francia.

Pero el amplio panorama de la fenomenología actual demanda al menos dos tipos de preguntas: en primer lugar, siendo tantas las propuestas fenomenológicas donde varían los fenómenos a describir (el acontecimiento, la carne, el ícono y el ídolo, la afectividad, etc.), es sensato preguntarse por los ajustes metódicos precisados, ajustes que dependen de los objetos a ser descritos. Mas, en verdad, ni siquiera se trata de "ajustes", pues el método mismo no puede ser sino la filosofía. Es, en este sentido, que las nuevas propuestas fenomenológicas no se desarrollan sin poner en cuestión el método de la manera más radical posible. Un buen ejemplo de ello es el último trabajo de Claude Romano (2010a), Au cour de la raison, la phénoménologie, donde el autor, reinterrogando la "experiencia", propone una revisión exhaustiva del método fenomenológico. Es así que el filósofo afirma:

Si hay algo que Husserl nos ha enseñado, es que el método no es importado desde el exterior en la filosofía, no consiste en un conjunto de preceptos vacíos dictados abstractamente, es decir independientemente de los objetos sobre los que trata esta disciplina, puesto que aquí el método es uno con la marcha filosófica misma y con la "cosa", el "asunto" (Sache) al que tiene por misión reconducirnos [...]. En fenomenología como en filosofía, el método es la cosa misma. (ROMANO, 2010a, p. 17).

Y un poco más adelante, agrega:

[...] es porque el presente libro es a la vez una introducción a la fenomenología (en el sentido de una introducción en la fenomenología, de una autopresentación de esta última) y una transformación de la fenomenología, la tentativa de una mejor justificación de sus tesis fundamentales -una presentación de la fenomenología a partir de ella misma en tanto que su propia transformación. (ROMANO, 2010a, p. 18).

Una segunda pregunta que puede plantearse es si es posible reconocer un hilo común entre las diversas propuestas fenomenológicas actuales a pesar de sus diferencias. Nuestra respuesta ante esta segunda cuestión es afirmativa. Pero antes de avocarnos a su justificación, es preciso constatar cómo la situación actual de la fenomenología viene a dar la razón al modo cómo Ricœur la presentaba ya en 1967 : 
[...] la fenomenología, dice el autor francés, es la suma de la obra husserliana y de las herejías resultadas de Husserl; es también, la suma de las variaciones de Husserl mismo y en particular la suma de las descripciones propiamente fenomenológicas y de las interpretaciones filosóficas por las que reflexiona y sistematiza el método. (RICCEUR, 2004, p. 9).

Treinta y nueve años después, Bruce Bégout, en un artículo sobre Paul Ricœur, comentando el texto recién citado, afirma que "[...] cada fenomenología avanza así por distancias y encauzamientos de los presupuestos iniciales de la fenomenología que remodela en función de temas y cuestiones que les son propias." (BÉGOUT, 2008, p. 197). Así también, Philippe Grosos (2014, p. 192) ha afirmado recientemente que

[...] lo propio de lo que se llama "fenomenología" es no existir en tanto que escuela o disciplina y tal vez probablemente ¡ni siquiera en tanto que tal! La fenomenología no existe sino para reinventar cada vez el modo de aproximación que es el suyo, para adaptarse mejor al fenómeno que intenta pensar. La única exigencia, si es que la hay, es pensar lo que se da en términos de fenómeno y por tanto pensar su modo de donación.

Y un poco más adelante agrega: "El despliegue de la fenomenología hay cada vez que reinventarlo en función de la cosa misma que hay que pensar. Radica allí su dificultad, pero igualmente su pertinencia y su interés." (GROSOS; VINCENT, 2008, p. 134).

El carácter herético de la fenomenología, tan plural y fructífero como sea, ha sido objeto de una polémica importante promovida por Dominique Janicaud (2009), quien ha acusado a las obras de Marion, Chrétien, Levinas y Henry, de haber dado un giro teológico. Recientemente, Isabelle ThomasFogiel califica a las fenomenologías actuales de un giro empírico o realista -sobre este giro volveremos a lo largo de la exposición-- y Christian Sommer de un giro antropológico. Mientras que Thomas-Fogiel (2013, p. 529) reconoce que la actual fenomenología "[...] profesa una forma de realismo de la experiencia que es el verdadero punto de unión (lugar de reparto) de las diferentes elaboraciones." Christian Sommer (2011, p. 160) afirma que

[...] lejos de ver en esta proximidad con la antropología un déficit, se podría encontrar allí la ocasión de un posible retorno al (del) "ser humano encarnado", "en carne y hueso", puesto entre paréntesis por la fenomenología trascendental como por la ontología fundamental. Lo que pide rehabilitar la antropología, interrogándose sobre su forclusión y su rechazo por parte de Husserl y Heidegger. 
Por su parte, Bruce Bégout ve un intento constante de la fenomenología practicada en Francia de anexarle a ésta -la fenomenología-- una doctrina "extra-fenomenológica" que viene a resistir al idealismo trascendental contra el cual la nueva generación se ha alzado. Al respecto, Bégout (2008, p. 132) afirma que:

Si los filósofos post-husserlianos separan a los hermanos siameses de la fenomenología y la filosofía trascendental es, a continuación, para anexarle a la parte fenomenológica otro cuerpo extraño: ontología, antropología, hermenéutica, filosofía de la vida, de la donación, del Otro. Apenas liberada del yugo del idealismo la fenomenología no tiene tiempo de gozar de su nueva libertad pues es asociada, incluso sujetada, a una nueva doctrina extra-fenomenológica para darle una base filosófica más sólida que la que le aportaría el idealismo trascendental.

Se comprende, por tanto, que ante la constante acusación que recae sobre la fenomenología practicada en Francia, de dar un giro o teológico, o antropológico, o empirista, lo que está en juego es la necesidad de reconocer una suerte de "topos común" (THOMAS-FOGIEL, 2013, p. 528) a los autores de esta última generación fenomenológica. Por mi parte, quisiera proponer brevemente algunos argumentos que permitan, por un lado, reconocer una preocupación común a la nueva fenomenología en Francia, tomando en cuenta dos corrientes fenomenológicas con orígenes distintos: por un lado, consideraremos las obras de Marion y Romano, que son más bien deudores de la filosofía heideggeriana y husserliana; y por otro lado, nos centraremos en la obra de Renaud Barbaras, más próximo a Husserl, Merleau-Ponty y Marc Richir. Siguiendo parcialmente los análisis de Isabelle Thomas-Fogiel, parece posible reconocer que una preocupación constante de la fenomenología actual es la donación; en este sentido, la obra de Renaud Barbaras, La dynamique de la manifestation (2013) -junto a Étant donné (2005) de Marion y L'événement et le monde (1998) de Romano--, viene tal vez a corroborar esta hipótesis de lectura a la que adhiero y que quisiera defender ${ }^{3}$. Un balance crítico con las obras de Marion y Romano puede aportar elementos suficientes para su justificación. Pero, para ello y así poder desplegar los argumentos a favor de la tesis del predominio de la donación en la práctica fenomenológica actual -y a modo de contraste--, quisiera presentar en primer lugar el modo cómo

\footnotetext{
${ }^{3}$ A pesar de que adhiero a la tesis de Isabel Thomas-Fogiel respecto del predominio de la cuestión de la donación en la nueva fenomenología en Francia, no comparto, sin embargo, la tesis general de su último libro, Le lieu de l'universel (2015), según la cual la actual fenomenología francesa habría dado un giro hacia una fenomenología realista. Cf. Mena (2015, p. 107-137).
} 
la fenomenología husserliana y heideggeriana fue recepcionada por la primera generación de fenomenólogos en Francia, a saber Sartre, Merleau-Ponty y Ricoeur.

2- "No es ni la exigencia de fundación última, ni la reivindicación de evidencia apodíctica de la conciencia de sí que fueron primero destacadas, dice Ricœur, sino al contrario, lo que en el tema de la intencionalidad rompía con la identificación cartesiana entre conciencia y conciencia de sí." (RICCEUR, 1995, p. 17). Con este texto, Paul Ricœur se refiere al impacto que causó la fenomenología en la primera generación de fenomenólogos franceses, a saber Emmanuel Levinas, Merleau-Ponty, Jean-Paul Sartre y él mismo. Es el descubrimiento de la intencionalidad lo que ha marcado de manera profunda los primeros desarrollos de la fenomenología en Francia, tal como puede atestiguarlo el breve ensayo de juventud dedicado por Sartre a la intencionalidad fenomenológica. Este descubrimiento venía, de todos modos, de la mano de la lectura de Sein und Zeit de Heidegger y su ruptura con la fenomenología husserliana, a excepción de Ricœur que era influido más por los existencialismos de Marcel y Jaspers, antes que por la ontología fundamental de Heidegger. No es posible entender el desarrollo de la fenomenología en las obras de Sartre, Merleau-Ponty, Ricœur y Levinas sin comprender que éstas toman su curso a partir del de las lecturas cruzadas entre Husserl y HeideggerEs así que Merleau-Ponty, en una entrevista publicada en 1946, explicaba que sus filosofías -las de Husserl y Heidegger- les aportaba a los fenomenólogos de su generación "[...] una filosofía extendida, un análisis sin prejuicios de los fenómenos, es decir del medio en el cual se desarrolla nuestra vida concreta." (MERLEAU-PONTY, 1997, p. 67). Se trataba, para Merleau-Ponty y Sartre, en opinión de Françoise Dastur, "[...] de encontrar en esta 'filosofía de la existencia' que les venía de Alemania, a través de Husserl y Heidegger, el medio para salir de la estrechez de una filosofía reflexiva de inspiración cartesiana y de pensar la situación concreta del hombre en el mundo y a través de la historia." (DASTUR, 2011, p. 37).

Por otro lado, es claro que esta primera generación de fenomenólogos franceses buscan tomar distancia del idealismo trascendental husserliano en fidelidad al lema que demandaba "[...] volver a las cosas mismas." (HUSSERL, 1985a, 218). Es así que el proyecto de una filosofía de la voluntad de Ricœur buscaba realizar una descripción eidética del acto voluntario -proyecto inspirado en la Phénoménologie de la perception de Merleau-Ponty que dejaba el campo de la vida práctica, a su juicio, sin explorar-. En Le volontaire et l'involontaire, Ricour, para llevar a cabo la eidética de la voluntad, se sitúa, descriptivamente 
desde la intencionalidad: siendo que como sujetos intencionales somos ciegos a la intención por la que estamos lanzados al mundo, no queda más que comenzar la descripción tomando primeramente en cuenta eso a lo que estamos vueltos intencionalmente; tal como lo afirma Ricœur (1950, p. 10), son "[...] las articulaciones de lo 'querido' como correlato del querer que orientan la descripción.” Y posteriormente, afirma:

Con Husserl llamamos intencionalidad a ese movimiento centrífugo del pensamiento vuelto hacia un objeto: yo soy en eso que veo, imagino, deseo y quiero. La intención primera del pensamiento no es atestiguarme mi existencia, sino unirme al objeto percibido, imaginado, querido. Si llamamos proyecto en sentido estricto al objeto de una decisión -lo querido, lo que decido- decimos: decidir es volverse hacia el proyecto, olvidarse en el proyecto, ser fuera de sí en el proyecto, sin detenerse a mirarse queriendo. (RICEUR, 1950, p. 42).

Hay aquí, al menos en la obra de Ricœur, el desarrollo de una fenomenología que se deja orientar por la objetividad y que impulsará posteriormente el injerto hermenéutico en la fenomenología que demanda un cogito capaz de dejarse instruir por los signos, los símbolos y los textos.

Si los caminos emprendidos por Merleau-Ponty, Sartre y Ricœur son diferentes, incluso contradictorios entre sí -mientras que Merleau-Ponty y Sartre rechazan todo tipo de reflexividad cartesiana, Ricœur adhiere a una reflexividad vuelta hermenéutica--, cada una de estas propuestas busca rechazar un cartesianismo ingenuo. Es por lo demás, la tarea actual de la fenomenología, a juicio de Claude Romano (2012). De este modo, si hay un punto coincidente entre la primera generación y aquella conformada por los trabajos de Marion y Romano, por tomar solo a estos, es la distancia asumida con respecto a la subjetividad trascendental. Pero esta vez, el camino es inverso. Mientras que la primera generación encontraba razones para oponerse al idealismo fenomenológico tomando como vía privilegiada para acceder a las cosas mismas a la intencionalidad, al correlato intencional; los trabajos de Marion y de Romano, por el contrario, resisten a la subjetividad trascendental invirtiendo la intencionalidad husserliana. Por un lado, mientras que la primera generación estaba en busca de lo originario o archi-originario (la carne para Merleau-Ponty, el otro para Levinas, etc.), la generación actual es más atenta a la multiplicidad de fenómenos dados. Un buen ejemplo de esto es el último libro de Jean-Yves Lacoste, Etre en danger, que en palabras de Isabelle Thomas-Fogiel (2013, p. 532) 
[...] denuncia la preocupación de una experiencia originaria y sus derivas hacia las temáticas de lo "fundamental", para oponerle una fenomenología "abierta", siempre capaz de ofrecer hospitalidad a nuevos fenómenos [...]. Se trata, en un tal cuadro, agrega Thomas-Fogiel extendiendo el análisis a las obras de Marion y Romano, de no hacer más que describir lo que se da, puesto que en Marion como en Romano o incluso en Richir (por su teoría de la "formación espontánea del sentido"), toda la iniciativa proviene del fenómeno que surge (Richir) o adviene (Romano).

Volver a las cosas mismas significa dejar que los fenómenos se den, esto es que se muestren en su aparecer por sí mismos y, por tanto, dejar al "si" del fenómeno manifestarse; entonces, lo que se conoce como el idealismo de la fenomenología trascendental se vuelve, ciertamente, problemático. Es, claramente, las Meditaciones cartesianas el lugar donde mejor se deja expresar esta tesis. En el parágrafo 33, Husserl (1985b, p. 123) realiza la siguiente declaración:

Como el ego concreto que es la mónada comprende la vida entera, real y potencial, de la conciencia, es claro que el problema de la exhibición fenomenológica de este ego-mónada (el problema de su constitución para sí mismo) ha de abarcar todos los problemas de constitución. Otra consecuencia es la identidad de la fenomenología de esta autoconstitución con la fenomenología en general.

Luego, en el parágrafo 41 agrega:

Llevada a cabo en esta concreción sistemática, es la fenomenología eo ipso "idealismo trascendental", bien que en un sentido radicalmente nuevo. No en el sentido de un idealismo psicológico, en el de un idealismo que se empeña en sacar de datos sensoriales sin ningún sentido un mundo con un sentido. Tampoco es un idealismo kantiano, que cree poder dejar abierta, al menos como concepto límite, la posibilidad de un mundo de cosas en sí; sino un idealismo que no es exactamente nada más que una autoexposición de mi ego en cuanto sujeto de todo conocimiento posible, y por respecto a todo sentido de realidad con que haya de poder haber para mí, el ego, un sentido, desarrollada esta exposición consecuentemente como una ciencia egológica sistemática. (HUSSERL, 1985b, p. 143).

Se entiende que lo propio de la tesis idealista de la fenomenología trascendental consiste en homologar la autoconstitución del ego-mónada con 
el problema de la constitución en general. Al respecto, Ricœur (2004, p. 229) afirma que:

El yo no es ya simplemente el polo sujeto opuesto al polo objeto, es el englobante: todo es Gebilde de la subjetividad trascendental, producto de su Leistung: la fenomenología es "Selbstauslegung meines ego, als subjekten jeder möglichen Erkenntnis". Es por tanto en la doctrina del ego y de su constitución temporal que se vinculan de modo inseparable fenomenología e idealismo.

Si el idealismo de la fenomenología consiste en reducir la explicitación del mundo a la del ego-mónada, entonces el fenómeno no solo es lo que se da "para mí", sino también "en mí" y "a partir de mí" (RICEEUR, 2004, p. 184), quedando oscurecida toda región de alteridad, en cuanto el fenómeno, cualquiera que sea, no se da sino gracias al poder constituyente de la subjetividad, no siendo sino un momento mismo del flujo mismo de la vida de la conciencia; bajo este respecto, el fenómeno es englobado por el ego trascendental. El ego-mónada no tiene como correlato ningún otro, ninguna alteridad que lo confronte, que se le imponga, porque todo lo dado, todo fenómeno dado no viene sino a explicitarlo a él mismo.

El reproche que le dirige la nueva fenomenología en Francia a la fenomenología en su caída en el idealismo trascendental, tal como es entendido por Husserl, es que este último olvida que siendo la conciencia la llamada a aclarar el sentido del fenómeno, este se da, sin embargo, por sí mismo y mantiene un ingrediente de alteridad insuperable porque inabordable de modo definitivo. Un fenómeno constituido en, para y a partir de la conciencia constituyente deja de ser propiamente un fenómeno, si es que su mostranza (mostrance, Erscheinung, tal como lo afirma Romano siguiendo a Claudel y a Hegel (ROMANO 1998, p. 42; GABILLIERI, 2015) destaca también la influencia hegeliana en el modo como Marion comprende el fenómeno como aquello que se da por sí sin condición) se confunde con la autoconstitución del ego trascendental.

A juicio de Isabelle Thomas-Fogiel -habría que agregar también a Jocelyn Benoist quien reconoce en la fenomenología "un cierto empirismo" en la referencia a lo dado (cf. BENOIST, 2001, p. 45)-, la máxima "volver a las cosas mismas" se ha transformado en un "volver de las cosas mismas" (THOMAS-FOGIEL, 2015, p. 39-50). No se trata de una traición a la consigna husserliana sino de su efectivo cumplimiento o concreción. "Volver 
a las cosas mismas requiere aprender a tomarlas en su aparecer y, por tanto, como fenómenos. Son estos últimos desde donde es preciso partir, siendo que, de algún modo, los fenómenos se imponen por sus propios medios (cf. MARION, 2005, p. 16) en tanto que su aparecer nos sobreviene (cf. DE GRAMONT, 2014, p. 141). ¿Cómo ser fiel a la máxima fenomenológica que nos pide volver a las cosas mismas, esto es a las cosas en su donación en persona, a su manifestarse por sí mismo? Esto, en tanto consideremos que fenómeno es, precisamente, "lo que se muestra a partir de sí mismo" ( HEIDEGGER, 1997, p. 51-55; ROMANO, 1998, p. 42; MARION, 2005, p. 13-17). Así, lo que está en cuestión es cómo y de qué manera "dejar" aparecer a las cosas mismas en su mostrarse, en su "mostranza", ya no englobadas y ensombrecidas por el ego trascendental.

Lo que se muestra por sí mismo, el fenómeno, es, al mismo tiempo, lo que guía y determina los modos de su aproximación con el fin de liberar su aparecer en tanto tal -la reducción mantiene, al menos en la filosofía de Marion, aún su lugar-. Afirma De Gramont (2014, p. 144):

Al comienzo, es el fenómeno, y la primera palabra del pensamiento es aquí para decir que viene en segundo lugar. En tanto que el impulso de la búsqueda no puede venir sino de las cosas mismas, entendidas en el sentido de lo que se muestra (fenómeno), lo que se da desde sí mismo (lo dado), o lo que viene a partir de sí mismo (el acontecimiento). La máxima husserliana que nos ordena ir "derecho a las cosas mismas" no dice otra cosa, y podemos recibir como libres variaciones sobre el mismo tema estos otros preceptos que nos piden "dejar la primacía al fenómeno" (y en consecuencia "dejar al fenómeno advenir él mismo"), o "pensar el acontecimiento antes de toda cosa”.

La máxima husserliana puede ser comprendida como una conminación a dejar advenir al fenómeno por sí mismo, al punto que éste termine por imponerse en su propio aparecer. No se recobra el mundo de la experiencia humana, ante le hegemonía del mundo de los objetos científicos que operan un hiato entre nuestras percepciones sensibles y el mundo vestido con ideas, sin que tal recuperación consista primariamente en aprender a recibir los fenómenos, en dejar que éstos se nos impongan antes que nosotros los constituyamos. Es también lo que ha indicado Jean Greisch (2002, p. 21) al decir que: 
El sufijo "-logía" no tiene el mismo sentido en el sintagma "fenomenología" que en los términos "biología" o "filología”. No se trata de mantener un discurso sobre los fenómenos, sino de escuchar la voz misma de los fenómenos. La fenomenología debe laboriosamente forjar un lenguaje que esté a la altura de la donación de los fenómenos, lo que implica una cierta violencia hecha al lenguaje ordinario, siempre más o menos cómplice de la actitud natural.

Lo que es puesto en juego, por las nuevas fenomenologías llevadas a cabo en Francia, las de Marion y Romano en este caso, es, por tanto, un intento por volver a pensar el proceder metódico mismo de la fenomenología: ésta no puede ser un idealismo trascendental, tal como Husserl llega a creerlo en las Meditaciones cartesianas, porque, si tal es el caso, lo que queda irremediablemente ensombrecido es la potencia misma del fenómeno, su ipseidad, vale decir el hecho de que éste aparece por sí mismo exceptuándose de toda condición previa que le fuera asignada para aparecer. A juicio de Marion, la fenomenología debe avanzar conforme al respeto escrupuloso por describir los fenómenos en su donación, en su mostración, siendo que nada “[...] hace excepción a la donación.” (THOMAS-FOGIEL, 2013, p. 530). De este modo, la fenomenología tiene que liberar al fenómeno de las condiciones que el sujeto, la subjetividad trascendental, le impone. Se trata de "[...] dejar a la aparición mostrarse en su apariencia según su aparecer.” (MARION, 2005, p. 13). Pero, ¿qué puede significar liberar al fenómeno de las condiciones que la subjetividad le impone? ¿No significa eso que lo que se da por sí mismo no se deja restringir ni constituir -al menos en el sentido idealista ya examinadopor el sujeto ni suscribe a ningún a priori? (DE GRAMONT, 2014b, p. 209). En efecto, el fenómeno propiamente tal, porta sus propias condiciones de posibilidad, siendo, en este sentido, incondicionado (ABELLIERI, 2015, p. 38). Así, propiamente, fenómeno es aquello que en su aparecer no reconoce límites que se le impongan por ningún tipo de subjetividad trascendental -ni kantiana ni husserliana-, apareciendo "por sî". Liberando al fenómeno de las condiciones impuestas por la subjetividad, se recobra el campo del aparecer mismo que excede, por lo demás, al de los entes y de los objetos. En palabras de Christian Sommer (2011, p. 152):

[...] es la donación, como figura fundamental de la fenomenalidad que permite liberarse del ideal de la representación objetivante, y el origen de la donación es precisamente el "sí" del fenómeno que no puede ser constituido por un ego trascendental, un "si" marcado por una determinación de acontecimiento: mostrándose, el fenómeno propiamente "inmirable" sobreviene de sí mismo, sin causa antecedente. 
3- Si la fenomenología se da por conminación el volver a las cosas mismas, lo hace, precisamente, porque éstas no nos son dadas de modo inmediato, de ahí el necesario recurso a la reducción, tal como Marion la comprende. Afirma Marion (2012, p. 122):

La filosofía tiende, bien entendido, a conocer las cosas mismas como ellas son, pero esta definición misma, "las cosas tales como son", constituye ya una operación. No se puede hablar de las cosas "tales como son", ni del "ente en tanto que ente", sin ejecutar una operación, puesto que se reduce lo que aparece a lo que es, o más bien a lo que es en él. La inmediatez aparente de la aparición misma resulta de una operación, no sería más que de una interpretación. La inmediatez no aparece -si puedo decirlo- más que si se suspende en ellas las mediaciones ya implícitas o utilizadas en un momento dado para producirla. También el retorno a las cosas mismas indica que éstas no son inmediatamente dadas, que su inmediatez debe ganarse mediatamente en la medida que es necesario precisamente volver a ellas por reconducción, reducción.

Este volver a las cosas mismas, en tanto que fenómenos, conlleva un examen de las operaciones mismas de la fenomenología. Pero no se trata de un mero asunto metodológico, sino, por el contrario: lo que está en cuestión es su proceder mismo, su marcha y encaminamiento hacia las cosas, por tanto, el método como orientación fundamental hacia éstas. La palabra de orden de la fenomenología, volver a las cosas mismas, plantea al fenomenólogo la cuestión relevante del examen del modo cómo acceder a éstas.

Que este acceso no sea inmediato es lo que ha constatado la fenomenología actual, tal como lo indicaba Marion, así como también lo ha hecho Romano -mas he aquí un disenso-:

[...] no hay jamás fenómenos que serían ofrecidos tal cual a una descripción, ninguna inmediatez de una donación de la que se podría esperar toda la luz: todo acceso a los fenómenos es irremediablemente mediato. Conviene renunciar al mito de un "puro dado", solidario aquel (cartesiano y husserliano) de una ausencia total de presuposiciones cuya descripción podría prevalerse. (ROMANO, 1999, p. 2-3).

Pero, si ambos textos, el de Marion y el de Romano, parecen indicar lo mismo: el mito de lo 'dado puro' e inmediato al que es preciso renunciar; al mismo tiempo, no es la misma vía la que emprenden ambas fenomenologías. Así, constatando el acceso mediato a los fenómenos, una, la fenomenología 
de la donación de Marion, privilegia la reducción como el proceder según el cual es posible determinar los "[...] grados y figuras de la donación, para no confundir a unos con otros, ni con otras instancias distintas a lo dado mismo" (MARION, 2012, p. 124); por su parte, la fenomenología del acontecimiento de Romano, se reconoce ella misma como una hermenéutica del advenir, sin tener que recurrir a la reducción.

La crítica que conduce Romano al recurso a la reducción, por parte de Marion, la ha expresado del siguiente modo: "[...] si vuestro fin es sacar a la luz el "si" del fenómeno y dejarlo a su propia iniciativa, liberarlo de toda instancia trascendental que aportara la medida de su fenomenalidad, ¿por qué hacer un llamado a un procedimiento [la reducción] que designa al método trascendental por excelencia?" (ROMANO, 2000, p. 11). La respuesta de Marion es clara. Tal objeción supone: 1) que se considere que la reducción conserva un estatuto trascendental "[...] adosándose a un $Y o$ él mismo incondicionado" (MARION, 2015, p. 218), mientras que el Yo que opera la reducción ha sido en verdad modificado por la reducción misma. Por lo que la "trascendentalidad" no se dice, necesariamente -y ese es el caso para Marion- del ego, sino de la reducción. Es decir, Marion postula una reducción sin un sujeto trascendental, por lo que el fenómeno, propiamente tal, no se da, no aparece ni se muestra por condición alguna impuesta por la subjetividad trascendental. Pues, la reducción a la donación (la tercera reducción que postula Marion, luego de la reducción al objeto -Husserl - y al ente - Heidegger) no es una operación llevada a cabo por un ego trascendental, sino por un sujeto ya reducido por la reducción misma; así, su objetivo no es sino dilucidar el "[...] grado de doneidad de este aparecer, hasta qué punto da y se da." (MARION, 2012, p. 127). Gracias a esta reducción a lo dado según su manifestación, Marion puede delimitar entre fenómenos saturados o paradojas, fenómenos pobres y de derecho común, siendo los primeros, los fenómenos saturados, los que propiamente se dan de modo incondicionado.

Tal como se puede apreciar en la objeción que Romano formula a Marion, el autor de L'événement et le monde opta por renunciar de modo definitivo a la reducción en fidelidad a la comprensión del fenómeno como aquello que se da por sí. En ambos autores hay, por tanto, un rechazo a sostener un ego trascendental que englobe, en su poder constituyente, al fenómeno reducido al punto que su explicitación no sea sino un momento de la explicitación del ego. Hay coincidencia en el respeto absoluto por dar cuenta del fenómeno en su capacidad de mostrarse por sí, incondicionado, liberado, por tanto, de las condiciones que impone el sujeto trascendental. 
Y al mismo tiempo, hay acuerdo en mantener aún lo trascendental; mientras que Marion lo dice de la reducción, Romano lo reconocerá en el darse $a$ posteriori del acontecimiento, que para él es el fenómeno insigne. No siendo nosotros contemporáneos de su advenimiento, del hecho de que nos arribe singularizándonos, sorprendiéndonos, portando una novedad que estremece nuestra aventura, éste, el acontecimiento, se nos da a la postre, après coup, demandando de nosotros, los advinientes, una comprensión que es siempre retrospectiva. De este modo, el no darse inmediatamente del fenómeno insigne no demanda operar la reducción, sino un ejercicio comprensivo retrospectivo del conjunto de posibles -en su totalidad- que ha sido remecido y transformado por el acontecimiento. Así, mientras que Marion requiere de la reducción para discriminar lo dado y sus modos de donación, Romano le asigna a la comprensión la tarea de dar mejor cuenta de los fenómenos que se interpretan a la luz del acontecimiento (ROMANO, 1998, p. 202-211).

En suma, la fenomenología de Marion, así como la de Romano, siendo fiel a la máxima husserliana de ir a las cosas mismas ha debido revisar aquello que llamamos fenómeno. El punto de apoyo se halla, a su juicio, en el parágrafo 7 de Ser y tiempo: fenómeno es lo que se muestra por sí mismo. Aquello implica una revisión de la marcha metódica de la fenomenología, sin la cual no es posible abocarnos pronto a la descripción de las esencias del fenómeno. En primer lugar, si fenómeno es lo que se muestra por sí, entonces es preciso renunciar a la instancia del Ego trascendental y, por consiguiente, de un sujeto fundante y constituyente: el sujeto es más bien constituido por el fenómeno que en su arribo lo afecta; el fenómeno que se da por sí, dándose al sujeto, se le impone al punto que éste se recibe gracias y a partir del fenómeno que se le da. Mas, es preciso aquí destacar entonces lo siguiente. Rechazando el Ego trascendental -y con ello a la fenomenología en su versión idealista-, se aboga por dejar aparecer al fenómeno por sí, esto es en toda su radical alteridad; no solo se pone en cuestión la instancia constituyente y englobante del ego-mónada, sino también el movimiento intencional por el cual el objeto es mentado. Dejar aparecer al fenómeno y aprender a recibirlo implica enfatizar y realzar su alteridad, que era lo que no permitía el idealismo trascendental en cuanto el fenómeno era un momento de la explicitación del ego.

Por otro lado, la vía fenomenológica de Marion confirma aún que el acceso a los fenómenos es posible a partir de una experiencia fenomenológica que se hace y que supone una serie de operaciones que hay que llevar a cabo para dejar que el fenómeno se de. De este modo, la fenomenología reivindica una experiencia que se hace $-\mathrm{y}$ no meramente que se tiene- en pos de dejar 
mostrarse aquello de lo que somos ciegos en la actitud natural, el fenómeno y sus procesos de constitución; es una experiencia en tanto relación o correlación. Husserl ha vuelto evidente que no se trata de pensar un polo con independencia del otro, sino que lo dado mismo es por y gracias a la relación entre ambos polos, ambos inseparables entre sí. Jean-Luc Marion reconoce el acceso mediato a los fenómenos y, con ello, reivindica aún la reducción como modo de acceso a estos; pero opera una reducción de los fenómenos a la donación, y no a la objetidad ni a la entidad. Mas, al mismo tiempo, propone una inversión de la vía intencional de la fenomenología, también realizada por Henry Maldiney, Claude Romano, y buena parte de la nueva fenomenología en Francia. Con ello, no niega ninguno de los dos polos de la correlación, sino que acentúa el movimiento que va del fenómeno al sujeto. El objetivo, común por ejemplo a Maldiney, es dar cuenta del aparecer de los fenómenos sin tener que reducirlos a objetos o entes; así, por ejemplo, Maldiney ante la pregunta “¿qué es el aparecer?” responde: "El aparecer, el phainesthai no tiene más acá. Él aporta y lleva consigo su comienzo. Lo que aparece se descubre por sí mismo a partir de nada." (MALDINEY, 2012, p. 16); Sobre Maldiney y la cuestión del aparecer, cf. (BARBARAS, 2014; ESCOUBAS, 2014; GROSOS, 2014; HOUSSET, 2014).

4 - Liberar el campo del aparecer de las condiciones impuestas por el sujeto constituyente, significa también operar una transformación profunda en la manera de comprender al sujeto a quien los fenómenos le advienen. A partir de Marion, se puede hablar de una subjetividad en "segunda instancia", es decir que se deja constituir por los fenómenos que arriban y su modo de manifestación. Es así que el filósofo francés dejará de hablar de sujeto para referirse más bien al adonné e interoloqué, es decir a aquel que se recibe de lo que se le da y de lo que lo llama; Claude Romano, por su parte, propondrá hablar del advenant ante los acontecimientos que le arriban y Jean-Louis Chrétien de un répondant capaz de responder a la palabra que nos interpela y demanda y que se deja escuchar en nuestras respuestas. De este modo, "[...] lo dado surge y se impone a mí sin que lo ordene y lo construya." (THOMAS-FOGIEL, 2013, p. 533). Bajo este respecto, el sujeto, antes que ser "espectador constituyente", es pensado como "testigo constituido" (cf. MARION, 2005, p. 302) del fenómeno que "avanza como 'inmirable', irreductible', 'inconstituible'” (SOMMER, 2012, p. 153).

El devenir testigo del sujeto conlleva, al mismo tiempo, una contraexperiencia que, antes de plantearnos ante un objeto, nos deja frente a 
una paradoja; se trata de una inversión de la intencionalidad, una contraintencionalidad o una intencionalidad invertida por los fenómenos saturados (donde la intuición del fenómeno da desmesuradamente más de lo que la intención podría tender y prever): se trata de una "[...] experiencia que resiste a la condiciones de objetividad.” (MARION, 2005, p. 300).

En el caso de la hermenéutica acontecial de Claude Romano, el advenant no impone tampoco condiciones de recepción al fenómeno del acontecimiento. Muy por el contrario, el arribo del acontecimiento provoca, por un lado, una reconfiguración del mundo del sujeto como también una crisis, una epojé historial. Del mismo modo, Henry Maldiney (2001, p. 92) afirmaba que:

Un acontecimiento es una desgarradura en la trama del ser-en-el-mundo, por tanto a la vez de la presencia y del mundo del que es el ahí. Y el acontecimiento es transformador. Se hace claro en la transformación a la que su integración nos obliga [...]. La irrupción del acontecimiento determina un estado crítico. Nuestra presencia en el mundo es amenazada, pues un acontecimiento no se produce en el mundo, es él al contrario que abre el mundo.

Es así que cuando un acontecimiento arriba, éste "[...] hace que yo no sea nunca más el mismo." (ROMANO, 2010b, p. 36; MICHEL, 2012, p. 46-53). No solo el advenant no pone las condiciones para la manifestación del acontecimiento, aunque sí podría decirse que él es la ocasión para su recepción pues solo hay acontecimiento para un sujeto capaz de hacer su experiencia, sino que además él mismo es configurado por su arribo: finalmente, para el advenant hacer la experiencia de los acontecimientos es hacer la de su propia transformación y devenir a raíz del arribo sorprendente, singularizante y epocal de los sucesos que nos advienen. ¿Por qué? Pues, al igual que Marion, Claude Romano intenta describir la manifestación de aquello que no se deja reducir a objetividad alguna. Es así que, en estricto rigor, ni tenemos experiencia de los acontecimientos -en el sentido de experiencia que nos reporte conocimientos acumulativos con bases objetivas--, ni hacemos su experiencia, si esto significa un encuentro con estos fundado en nuestras capacidades para reducirlo a algún tipo de objeto apreciable y manipulable. Por el contrario, el acontecimiento arriba siempre a nuestras espaldas pues su experiencia es la de la novedad absoluta, por lo que, rigurosamente a juicio de Romano, no somos sus contemporáneos. Dice Romano (1998, p. 196): 
La experiencia es ese riesgo de una exposición a eso que me alcanza en pleno corazón: por el acontecimiento que, destinándose a mí insustituiblemente, me da solamente el advenir a mí mismo [...]. Si la experiencia es desde entonces pensada radicalmente como travesía y ese riesgo en que estoy en juego yo mismo, a riesgo de perderme, no hay entonces experiencia en el sentido propio del acontecimiento [...]. La experiencia es esta travesía hacia sí a riesgo de sí, en tanto que exposición a todo otro: al acontecimiento.

Y si los acontecimientos, en tanto que nos afectan, nos tocan y nos son contingentes, suponen de parte del advenant la capacidad de recibirlos, ésta consiste en un poder qua pasividad: su poder es dado por los acontecimientos y no por el sujeto: se trata, por tanto, de la pasibilidad. "Pasible' significa 'capaz de padecer, de sufrir'; y esta capacidad implica una actividad, inmanente a la experiencia, que consiste en abrir su propio campo de receptividad" (MALDINEY, 2007, p. 265). ¿Una recepción antes de toda recepción ? Sí, pues no hay posibilidad de ninguna hospitalidad sin haber recibido, incluso a nuestra pesar. El camino de la recepción de los acontecimientos es trazado por ellos mismos; y el sujeto que los padece, solo puede responderles después de haberlos recibido.

En suma, el sujeto -que ya no puede ser pensado en tanto subjectum o hypokeimenon- es pensado en su capacidad para acoger y recibir el fenómeno -el acontecimiento, la llamada- y dejarse constituir por su arribo. Se trata de un sujeto que, sin haber perdido su cualificación de agente, se recibe a sí mismo por la novedad que portan los acontecimientos. Aún está en juego un sujeto libre y responsable, pero entendiendo que tal responsabilidad no queda limitada a la imputación de nuestras acciones y sus efectos que yo mismo y otros me pueden dirigir: hay aquí otro tipo de imputabilidad que es más bien señalada a partir del sentimiento de incumbencia. En efecto, los acontecimientos en cuanto traen consigo un sentido nuevo capaz de reconfigurar el todo de nuestra orientación, nos ponen en situación de responder de aquellos posibles a los que quedamos abiertos; la ipseidad, afirmará Romano, es precisamente aquella capacidad de responder de los acontecimientos; pero, es claro que no se responde de ellos, sino en cuanto que el sentido que nos propone, por el cual se reconfigura nuestro mundo, nos incumbe, sentimos o creemos que nos incumbe. De este modo, el sujeto, pasible ante los acontecimientos, responde de ellos en la medida que se reconoce incumbido por su sentido.

5- Hasta aquí el principal objetivo ha sido mostrar cómo la fenomenología actualmente practicada en Francia, principalmente en los trabajos de Marion 
y Romano, ha operado un giro con respecto a la primera generación, que hizo la recepción de la fenomenología husserliana y heideggeriana celebrando el descubrimiento de la intencionalidad; la generación actual ha tomado como punto de partida y de corrección metódica a la donación, lo que ha conllevado también, por un lado, la destitución del polo ego en su poder constituyente, y por otro, el examen de una contra-intencionalidad y, por tanto, el abandono de la objetividad como horizonte gnoseológico.

Ante este panorama, cabe preguntarnos qué lugar ocupa la obra de Barbaras en la fenomenología actual. Pues, incluso, si pueden reconocerse intenciones comunes entre las filosofías de Barbaras y de Marion -por nombrarlos solo a ellos--, también es indudable que la obra del autor de $\mathrm{La}$ dynamique de la manifestation (2013) representa la otra punta de la cuerda, siendo la primera aquella representada por Marion, Romano, Maldiney, etc. Las diferencias entre ambos movimientos fenomenológicos ¿se podrían expresar diciendo que mientras que aquel ha vuelto su mirada a la donación, aquel otro se ha dirigido hacia la vida? Tal vez se pueden reconocer algunos puntos comunes que atestiguan que, tratándose de dos polos de una misma cuerda, ambos están ligados en lo esencial. En primer lugar, la renuncia al subjetivismo trascendental y al ideal de intuitividad. En palabras de Pierre Rodrigo (2013, p. 256):

Es por tanto porque Husserl presupone que toda esencia es una plenitud de ser, dicho de otro modo un en sí según un modelo implícito que no es otro -importa notarlo- que el de la objetividad, que no puede pensar la intencionalidad de otro modo que como un mentar todo tendido hacia el ideal de intuitividad: a la esencia en tanto que plenitud de ser corresponde en la correlación una conciencia que tiende también a la plenitud por su exigencia de intuición.

Si este texto nos permite agregar razones para comprender por qué algunos de las fenomenologías actuales son contra-intencionales, otorgando un certificado de defunción a la subjetividad trascendental, en pos de un "sujeto" en segunda instancia que se recibe de lo que se dona, es preciso destacar que la fenomenología de Renaud Barbaras no deja de privilegiar "el a priori universal de correlación”, sin que ello signifique una vuelta atrás hacia un ideal de intuitividad ni hacia una reificación de la conciencia (BARBARAS, 2012). El énfasis que pone Barbaras en el a priori correlacional tiene por función salvar al aparecer de la reducción a un tipo particular de apareciente, tal como son las vivencias. "Porque, reconociendo el aparecer a las vivencias - hyléticas y noéticas- en el cuadro de una constitución trascendental, se 
subordina el aparecer a un cierto apareciente, a esta categoría singular de entes que son las vivencias, dándose así el aparecer de antemano en el momento en que se pretende dar cuenta de él." (BARBARAS, 2012, p. 51). De este modo, a diferencia de las fenomenologías de Marion y Romano, Barbaras no requiere postular una contra-intencionalidad para describir la donación de los fenómenos -en su más amplio espectro, como lo querría Jean-Yves Lacoste--, sino que le parece que, además de mantener y pensar el a priori correlacional, incluso llegando a aquellas consecuencias como las de renunciar a la reificación de la conciencia y la del ideal de intuitividad y que el propio Husserl no habría sabido vislumbrar, es lo que permite pensar verdaderamente la "autonomía de la manifestación", lo que significa que "[...] ella es independiente frente a todo apareciente, que no es de ningún modo tributaria de lo manifestado. Pues, lo propio de eso que se manifiesta en la manifestación es que su modo de ser es el de la cosa o, más precisamente, del ente." (BARBARAS, 2011a, p. 333).

La autonomía de la manifestación conlleva, por consiguiente, una crítica a las pretensiones de fundar el aparecer en la subjetividad, pues más que ser ésta constitutiva del aparecer de los entes, si tenemos en cuenta el $a$ priori correlacional, habría que decir que la subjetividad es una dimensión constitutiva del aparecer, y que éste mismo la hace posible "[...] como surgimiento de este ente singular que es la vivencia." (BARBARAS, 2011a, p. 334). Más que decir que el aparecer es subjetivo, cuestión también rechazada por las fenomenologías de Marion y de Romano, habría que afirmar con Barbaras que es una estructura que "[...] puede dar lugar al aparecer subjetivo." (BARBARAS, 2011a, p. 334). Pero, también hay que afirmar que la manifestación se mantiene autónoma frente al otro polo de la correlación, la objetividad. De este modo, Barbaras afirma que la manifestación no es cosa, no es objeto. Se trata, por el contrario, de un acontecimiento, el acontecimiento de la manifestación. Aquí podrían esbozarse algunos vínculos con la filosofía de Marion, que hace del fenómeno saturado precisamente un fenómeno que excediendo en intuición se dona como lo inmirable, lo inobjetualizable, lo intematizable. Pero he aquí otro punto de discusión: si tal como lo afirma Barbaras, la manifestación, sin ser cosa alguna, no puede advenir sino como cosa, entonces lo que queda al descubierto a partir del a priori correlacional es lo que el autor ha llamado la "dinámica de la manifestación" que no apunta sino al aparecer mismo de las cosas, a su movimiento de manifestación, a su develamiento y descubrimiento: "[...] manifestarse es ser descubierto, afirma Barbaras, pero ser descubierto es ser delimitado." (BARBARAS, 2011a, 344). Es lo propio del movimiento descubrir circunscribiendo, delimitando. Es la razón por la que el movimiento de la manifestación no produce el movimiento de los entes, sino que lo presupone. Pensar de este modo la correlación es 
dar cuenta de lo que el autor ha llamado estructura: ni el polo ego, ni la objetidad, sino la estructura misma que los reúne; es decir, el movimiento, el devenir, esto es ni substrato óntico ni determinaciones, sino el proceso o advenimiento de las cosas: "No hay ser maduro más que como movimiento de maduración, y es ese movimiento que produce conjuntamente la unidad de la determinación e identidad del substrato, o aún el ser-junto y el lugar que delimita." (BARBARAS, 2011a, p. 346).

El movimiento no puede ser sino un movimiento de limitación. Es así que el movimiento natural del mundo se da en cada ente limitándose a ese ente en particular, sin que aquel ente agote el movimiento mismo del mundo, y sin que el ente mismo finiquite aquellas manifestaciones, pues precisamente su movimiento o devenir consiste también en quedar abierto a otros modos de manifestación. Lo que está en juego aquí no es sino una negatividad fundamental que Barbaras (2012, p. 56) expresa del siguiente modo:

[...] se puede decir indiferentemente que lo finito es negación de lo infinito, puesto que toda aparición reenvía a un mundo que limita, y que lo infinito es negación de lo finito en tanto que el mundo no se revela más que en sus apariciones como lo que las excede. En una suerte de inversión intencional fundamental, la aparición hace aparecer al mundo como eso de lo que forma parte, aclara ante ella el mundo del que proviene sin embargo. El mundo ya no precede a la aparición como aquella no lo precede: uno y otro nacen juntos del dinamismo originario del aparecer.

El caso de la percepción es ejemplar: se puede afirmar, en cierto respecto, que la cosa es ya el esquema perceptivo mismo en cuanto su aparecer es por escorzos, no siendo los escorzos sino los modos cómo la cosa se da; aunque, por otro lado, los escorzos demandan ser superados hacia la cosa misma que, sin embargo, como se decía, no se da sino por perfiles. Si consideramos las cualidades de las cosas, como el ser rojo de tal o cual objeto, es al mismo tiempo experienciado como una "potencia inagotable de ser" (GÉLY, 2013, p. 247), es decir como una limitación del mundo, pero también como "[...] la presencia misma de una ausencia radical, de una ausencia de nada determinado.” (GÉLY, 2013, p. 247). Es así que en la percepción hacemos la experiencia tanto de una presencia como de una ausencia. Es este juego de presencia y ausencia, inseparables entre sí, que nos permite comprender que la percepción es experiencia de un exceso, de una nada que no puede ser determinada. Es como si cada cosa dada, dándose, manifestándose del único modo que puede hacerlo, mientras se da de ese modo, remitiera al mismo tiempo, porque en tanto movimiento queda abierta, a otros múltiples modos 
de manifestaciones que, ausentes, se hacen presentes como carencia subrayada por el aparecer de la cosa que no es sino limitación, esencia.

Ahora bien, este exceso no da cuenta sino de una no-coincidencia fundamental e insuperable entre el ente que aparece y sus apariciones. En palabras de Barbaras: "[...] el ente que aparece difiere de sus apariciones sin por ello ser otro que ellas.” (BARBARAS, 2011b, p. 159). ¿No encontramos aquí alguno de los argumentos con los que Barbaras busca escapar a la trampa de la reificación de la conciencia? Precisamente, la no-coincidencia del aparecer -lo que aparece y sus apariciones--, es signo también de una negatividad que, por un lado, es indeterminación y por otro negación de sí, en suma, movimiento. He aquí el último punto que me interesa subrayar; pues la fenomenología de Renaud Barbaras, siendo que se ha dado por tarea pensar con radicalidad el $a$ priori correlacional, termina por subrayar la relación necesaria y fundamental que el sujeto mantiene, sin poder sustraerse de ella, con lo otro para existir: "[...] su ser no reside en él mismo, en suma es caracterizado por una falta de ser [...]. Eso significa que el movimiento por el cual me parece deber definir el sujeto no es un movimiento cualquiera: su dinamismo será ordenado por una búsqueda de sí en lo otro, será aspiración.” (BARBARAS, 2011b, p. 162). En este sentido, el sujeto, el destinatario de la manifestación, no se comprende sino en y a partir del movimiento incesante que es su existencia, movimiento hacia el mundo que está caracterizado por ser indefinido: movimiento, por tanto, que no encuentra ni término ni reposo, que no deja de realizarse porque no termina nunca de cumplirse. Movimiento, por consiguiente, que es deseo y distancia.

El deseo tiene en efecto esto de propio que si algo puede satisfacerle nada puedo colmarlo, de modo que renace en lo que lo apacigua, como lo deseado exacerba en la medida que lo satisface. Todo pasa como si [...] la vida se confundiese con una aspiración a la venida del mundo. El sujeto existe como deseo del mundo, es decir como una aspiración que, a la vez, es susceptible de realizarse, precisamente, bajo la forma de la aparición de los entes finitos, pero no puede por principio ser colmada en tanto que el mundo se retira siempre de las apariciones en las que se presenta. (BARBARAS, 2012, p. 58).

El deseo es, bajo este respecto, movimiento hacia lo que el sujeto no es; movimiento que es principio de manifestación de las cosas. La negatividad propia de la manifestación, que no es sino la de la vida, tiene su fundamento en el deseo de ser más, de ser otro.

Para concluir, quisiera tan solo realizar una breve nota. Hasta aquí 
hemos intentado mostrar que la fenomenología de Barbaras toma lugar en la fenomenología actual desarrollada en Francia, renunciando por un lado al subjetivismo trascendental y, por otro lado, proponiendo una descripción del aparecer, tal como a su modo lo han hecho Marion y Romano. Pero quisiera, también, subrayar brevemente una cierta comunidad de pensamiento o, al menos un aire de familia, entre la propuesta de la fenomenología de la manifestación, de la dinámica de la manifestación, de Barbaras y la fenomenología hermenéutica de Paul Ricœur. Ciertamente, reconozco el abismo que separa ambas propuestas filosóficas, pero al mismo tiempo no se puede negar el interés común entre los dos filósofos por cuestiones como el deseo, el esfuerzo y el movimiento. Si para Barbaras el deseo muestra porque mueve, manifiesta porque es negatividad, para Ricœur el deseo también conlleva un movimiento y una negatividad, pues es deseo de ser y de persistir en la existencia (VALLÉ, 2013). Al respecto, Ricœur (1969, p. 95) afirma que: "La existencia [...] es deseo y esfuerzo. Nosotros la llamamos esfuerzo para señalar su energía positiva y dinamismo, la llamamos deseo para designar su carencia e indigencia." Incluso si el deseo es deseo de sí, incluso en dicho caso siempre se trata de un movimiento hacia una alteridad, movimiento mismo que manifiesta la relación estricta entre el sujeto y lo otro, y por tanto aquella distancia que, insalvable, no deja de ser al mismo tiempo un modo de proximidad. Pero decir proximidad es subrayar la distancia insalvable que no se deja suprimir jamás. La proximidad es distancia y, en ese sentido, el deseo es negatividad pues jamás se colma, jamás se detiene siendo movimiento, el movimiento del deseo, una suerte de perennidad insalvable.

MALET, Patricio Mena. Donation and subjectivity in the new French phenomenology: Marion, Romano and Barbaras. Tans/form/ação, Marília, v. 40, n. 1, p. 187-210, Jan./Mar., 2017.

\begin{abstract}
The aim of this paper is to examine the new phenomenological tendencies in France through the works of Marion, Roman, and Barbaras. Our hypothesis is that these phenomenologies, on the one hand those of Marion and Romano, and on the other hand that of Barbaras, agree on two main points: 1) the priority given to donation and 2) the need to think about subjectivity by putting aside Husserl's paradigm of transcendental subjectivity. We suggest that both philosophical approaches require an examination of Husserl's methodological pretensions, because it is in this context that the originality of their own phenomenological ideas can be noticed.
\end{abstract}

Keywords: Subjectivity. Donation. Desire. Event. Phenomenon. 


\section{REFERÊNCIAS}

BARBARAS, R. Introduction à une phénoménologie de la vie. Paris: Vrin, 2008.

. La phénoménologie comme dynamique de la manifestation. Les Études Philosophiques, v. 3, n. 98, p. 331-349, 2011 a.

. Sauver d'une réification de la conscience la tâche de la phénoménologie. Les Études Philosophiques, v. 1, n. 100, p. 49-63, 2012.

. La dynamique de la manifestation. Paris: Vrin, 2013.

. et al. La phénoménologie et le concept de vie: un entretien avec Renaud Barbaras. Journal of French and Francophone Philosophy. Revue de la Philosophie Française et de Langue Française, v. 19, n. 2, p. 153-179, 2011 b.

. L'essence de la receptivité: transpasibilité ou désir? In: VV. AA. Maldiney, une singulière présence. Paris: Encre Marine, 2014. p. 15-31.

BÉGOUT, B. Le phénomène et son ombre. Chatou: La Transparence, 2008.

DASTUR, F. Réception et non-réception de Heidegger en France. Revue Germanique Internationale: Phénoménologie Allemande, Phénoménologie Française, n. 13, p. 35-57, 2011.

DE GRAMONT, J. L'entrée en philosophie: les premiers mots. Paris: L'Harmattan, 1999. (Ouverture philosophique). . Au commencement: parole, regard, affect. Paris: Cerf, 2013. (La Nuit Surveillée). . L'appel de la loi. Louvain-Paris: Éditions de l'Institut Supérieur de Philosophie Louvain-la-Neuve; Éditions Peeters, 2014a.

. Portrait(s) de Jean-Luc Marion”. In: SOMMER, C. (Org.). Nouvelles

phénoménologies en France. Paris: Hermann, Rue de la Sorbonne, 2014b. p. 205-213.

ESCOUBAS, E. Henry Maldiney avec Dominique Janicaud: la résistance phénoménologique à la philosophie première et à l'ontho-théologique". In: SOMMER, C. (Org.). Nouvelles phénoménologies en France. Paris: Hermann, Rue de la Sorbonne, 2014. p. 115-126.

FALQUE, E. Passer le Rubicon: philosophie et théologie: essai sur les frontières. Bruxelles: Lessius, 2013. (Donner Raison, Philosophie).

. Le Combat amoureux: disputes phénoménologiques et théologiques. Paris:

Hermann, 2014. (De Visu).

GABELLIERI, E. Paradoxe, univocité, analogie. In: CAPPELLE-DUMONT, P. (Org.). Philosophie de Jean-Luc Marion: phénoménologie, théologie, métaphysique. Paris: Hermann, Rue de la Sorbonne, 2015. p. 31-48.

GÉLY, R. La finitude radicale de la vie: réflexions à partir de la phénoménologie de Renaud Barbaras. Alter: Revue de phénoménologie, v. 21, n. 21, p. 100-116, 2013. (La vie). 
GREISCH, J. Le buisson ardent et les lumières de la raison. L'invention de la philosophie de la religion. Tome II: Les approches phénomènologiques et analytiques. Paris: Cerf, 2002.

. Qui sommes-nous? Chemins phénoménologiques vers l'homme (Novembre 2006). Louvain-Paris: Éditions de l'Institut Superieur de Philosophie Louvain-la-Neuve; Éditions Peeters, 2009.

GROSOS, P. Henry Maldiney, Michel Henry et la critique de la phénoménologie. In: DE GRAMONT, J.; GROSOS, P. (Org.). Henri Maldiney: phénoménologie, psychiatrie, esthétique. Rennes: PUR, 2014. p. 13-22.

.; VINCENT C. Entretien avec Philippe Grosos. Le philosophoire. v. 2, n. 30, p. 127-145, 2008.

HOUSSET, E. Personne et sujet selon Husserl. Paris: PUF, 1997. (Épiméthée).

. Husserl et l'énigme du monde. Paris: Le Seuil, 2000. (Points, essais).

. La vocation de la personne: l'histoire du concept de personne de sa naissance augustinienne à sa redécouverte phénoménologique. Paris: PUF, 2007. (Épiméthée).

. L'anthropologie au risque de la phénoménologie dans Penser l'homme et la folie. In: DE GRAMONT, J.; GROSOS, P. Henri Maldiney: phénoménologie, psychiatrie, esthétique. Rennes: PUR, 2014. p. 53-73.

. La crise des sciences européene. Paris: Gallimard, 1976.

Investigaciones lógicas, I. Madrid: Alianza, 1985a.

. Meditaciones cartesianas. Madrid: FCE, 1985b.

. Philosophie première. 2. Théorie de la réduction phénoménologique. Paris: PUF, 1990. (Épiméthée).

JANICAUD. D. Le tournant théologique de la phénoménologie française In: La phénoménologie dans tous ses états. Paris: Gallimard, 2009. p. 39-149 (Folio Essais).

MALDINEY, H. Existence : crise et création . In: DE GRAMONT, J.; GROSOS, P. (Org.). Henry Maldiney entouré de ses amis. Existence. Crise et création. La Versanne: Encre Marine, 2001. p. 73-112.

. Penser l'homme et la folie. Grenoble: Million, 2007.

. L'art, l'éclaire de l'être. Paris: Cerf, 2012.

MARION, J.-L. Étant donné. Paris: PUF, 2005.

MENA, P. El fenómeno de la apelación. Co-herencia: Revista de Humanidades, Universidad EAFIT, v. 12, n. 23, p. 107-137, jul.-dic. 2015.

MERLEAU-PONTY, M. Parcours 1933-1951. Lagrasse: Verdier, 1997.

. La prose du monde. In: Euvres. Paris: Gallimard, 2000. (Quarto).

MICHEL, J. Sociologie du soi. Paris: PUR, 2012. 
RICEUR, P. Le volontaire et l'involontaire. Paris: Aubier, 1950.

RICEUR, P. Le conflit des interprétations. Paris: Seuil, 1969. Réflexion faite: autobiographie intellectuelle. Paris: Esprit, 1995. . À l'école de la phénoménologie. Paris: Vrin, 2004.

RODRIGO, P. Lacune et totalité: phénoménalité, vie et monde dans la pensé de Renaud Barbaras. Alter: Revue de Phénoménologie, v. 21, n. 21, p. 255-266, 2013. (La vie).

ROMANO, C. L'événement et le monde. Paris: PUF, 1998. . Au cour de la raison, la phénoménologie. Paris: Gallimard, 2010a. . L'aventure temporelle. Paris: PUF, $2010 \mathrm{~b}$. 100, 2012. . La phénoménologie doit-elle demeurer cartésienne? Les études philosophiques, n. . Le don, la donation et le pardoxe. In: CAPPELLE-DUMONT, P. (Org.). Philosophie de Jean-Luc Marion: phénoménologie, théologie, métaphysique. Paris: Hermann, Rue de la Sorbonne, 2015. p. 11-30.

SAUDAN, A. Penser Dieu autrement: introduction à l'œuvre d'Emmanuel Falque. Paris: Germina, 2013.

SOMMER, C. Le sujet sans subjectivité: après le "tournant théologique" de la phénoménologie française”. Revue Germanique Internationale: Phénoménologie Allemande, Phénoménologie Française, n. 13, p. 149-162, 2011.

TENGELYI, L. L'expérience de la singularité. Paris: Hermann, 2014. (Le Bel Aujourd'hui). .; GONDEK, H-D. Neue phänomenologie in Frankreich. Berlin: Suhrkamp, 2011.

THOMAS-FOGIEL, I. La tournure empiriste de la phénoménologie française contemporaine. Revue Philosophique, n. 4, p. 527-548, 2013.

- Le lieu de l'universel: impasses du réalisme dans la phénoménologie contemporaine. Paris: Le Seuil, 2015. (L'ordre Philosophique).

VALLÉ, M.-A. Ricœur et Spinoza: intérêt et difficultés d'une réappropriation herméneutique de la pensée de Spinoza. Alea. Revista internacional de fenomenología y hermenéutica, n. 11, p. 89-107, 2013.

Recebido em 29/08/2016

Aceito em 23/12/2016 